\title{
Sur une condition pour qu'un continu soit une courbe jordanienne.
}

\author{
Par \\ Wa cław Sierpiński (Warszawa).
}

Le but de ce Mémoire est la démonstration du suivant

Théorème. Pour qu'un continu $O$ (situé dans un espace euclidien à $m$ dimensions) soit une courbe jordanienne, il faut et il suffit que, pour tout $\varepsilon>0$, il soit une somme d'un nombre fini de continus de diamètre $<\varepsilon$.

Lemme I. Si un continu $C$ (dans l'espace à $m$ dimensions) est une somme d'un nombre fini de continus $A_{1}, A_{2}, \ldots, A_{s}$, chaque couple $A_{p}, A_{q}$ de ces continus peut être liè par une chaîne finie de continus

$$
B_{0}, B_{1}, \ldots, B_{r}
$$

telle que

$$
B_{0}=A_{p}, \quad B_{r}=A_{q},
$$

$$
B_{i} B_{i+1} \neq 0 \quad \text { pour } \quad i=0,1, \ldots, r-1,
$$

tout $B_{i}$ (où $i=0,1, \ldots, r$ ) étant un $A_{k}$ (où $k=1,2, \ldots, s$ ).

Démonstration. Soit donné un indice $p \leqslant s$. Désignons par $\mathfrak{M}$ l'ensemble de tous les continus $A_{q}(q=1,2, \ldots, s)$ pour lesquels il existe au moins une chaîne (1) satisfaisant aux conditions (2) et (3) (tout $B_{i}$ étant un $A_{k}$ ), et par $\mathfrak{N}$ l'ensemble de tous les autres continus $A_{q}$.

Admettons que l'ensemble $\mathfrak{N}$ n'est pas vide (l'ensemble $\mathfrak{N}$ n'est pas vide, puisqu'il contient $A_{p}$ ). Soient: $S$ la somme de tous les continus appartenant à $\mathfrak{M}$ et $T$ celle de tous les continus appartenant à $\mathfrak{N}$; les ensembles $S$ et $T$ sont évidemment 
fermés, comme sommes d'un nombre fini de continus. Or, on voit sans peine que $S$ et $T$ sont sans point commun. Supposons, en effet, que $x$ soit un point commun à $S$ et $T$. $n$ existe donc un $A_{h}$ dans $\mathfrak{M}$ et un $A_{j}$ dans $\mathfrak{\Re}$, tels que $x$ est un point de $A_{h}$ et de $A_{j}$. Nous arons done $A_{h} A_{j} \neq 0$; or, $A_{h}$ appartenant à $\mathfrak{M}$, il en résulte immédiatement, d'après la définition de $\mathfrak{M}$, que $A_{j}$ appartient aussi à $\mathfrak{N}$, contrairement à l'hypothèse que $A_{j}$ appartient à $\Re$. Les ensembles $S$ et $T$ sont done sans point commun. Il en résulte que le continu $C$ peut être décomposé en une somme $C=S+T$ de deux ensembles fermés sans points communs, ce qui est impossible. L'ensemble $\mathfrak{N}$ est donc vide, ce qui démontre notre lemme.

En particulier, lorsque $p=q$, on peut poser $B_{0}=A_{p}$ avec $r=0$.

Lemme II. Dans les hypothèses du lemme I, il existe pour $A_{p}$ et $A_{q}$ une chaîne (1) satisfaisant aux conditions (2) et (3) et qui contient (au moins une fois) tout continu $A_{k}(k=1,2, \ldots, s)$.

Démonstration, Appelons une chaîne (1) satisfaisant aux conditions (2) et (3) chaîne $\left(A_{p}, A_{q}\right)$ et écrivons consécutirement les termes des chaînes:

$$
\left(A_{p}, A_{1}\right),\left(A_{1}, A_{2}\right)\left(A_{2}, A_{3}\right), \ldots,\left(A_{s-1}, A_{s}\right),\left(A_{s}, A_{q}\right) .
$$

La chaîne ainsi obtenue satisfait évidemment à la thèse du lemme II.

Lemme IIT. Si l'ensemble $S$ est une somme d'un nombre fini de continus: $S=C_{1}+C_{2}+\ldots+C_{n}$, et si $S$ contient un continu $C$ tel que $C C_{i} \neq 0$ pour $i=1,2, \ldots, n$, l'ensemble $S$ est un continu.

Démonstration. Supposons que l'ensemble $S$ soit une somme de deux ensembles fermés non vides sans point commun: $S=A+B$. On voit sans peine que tout sous-continu $K$ de $S$ est contenu soit dans $A$, soit dans $B$. En effet, supposons que nous ayons simultanément $K A \neq 0$ et $K B \neq 0$; la formule $K=K A+K B$ donnerait alors une décomposition du continu $K$ en une somme de deux ensembles fermés sans points communs, ce qui est impossible. Nous avons donc soit $K A=0$, soit $K B=0$, ce qui prouve, d'après l'hypothèse $K \subset S=A+B$, que l'on a soit $K \subset A$, soit $K \subset B$. 
En particulier, en posant $K=C$, nous en concluons que le continu $C$ est contenu soit dans $A$, soit dans $B$, p.ex. $C C A$. Comme $C C_{i} \neq 0$ pour $i=1,2, \ldots, n$, on a donc $A C_{i} \neq 0$ pour $i=1,2, \ldots, n$. D'autre part, en posant $K=C_{i}$, nous concluons que tout $C_{i}$ est contenu soit dans $A$, soit dans $B$, d'où, vu que $A C_{i} \neq 0$ et $A B=0$, on a $C_{i} \subset A$ pour $i=1,2, \ldots, n$. Par conséquent, l'ensemble $S=C_{1}+C_{2}+\ldots+C_{n}$ est contenu dans $A$, contrairement à la supposition que $B \neq 0$.

L'ensemble $S$ ne peut donc pas être une somme de deux ensembles fermés non vides $A$ et $B$ sans point commun. Or, comme somme d'un nombre fini de continus, l'ensemble $S$ est fermé et contient plus qu'un point. L'ensemble $S$ est donc un continu, c. q.f.d.

Passons maintenant à la démonstration du théorème.

Supposons que le continu $C$ soit, pour tout $\varepsilon>0$, une somme d'un nombre fini (dépendant de $\varepsilon$ ) de continus de diamètre $<\varepsilon$. Nous pouvons donc écrire en particulier (pour $\varepsilon=1 / 2$ ):

$$
C=A_{1}+A_{2}+\ldots+A_{s},
$$

où $A_{1}, A_{2}, \ldots, A_{s}$ sont des continus de diamètre $<1 / 2$.

D'après le lemme II, il existe une suite

$$
C_{0}, C_{1}, C_{2}, \ldots, C_{m_{1}-1}
$$

telle que

$$
C_{i-1} C_{i} \neq 0 \quad \text { pour } \quad i=1,2, \ldots, m_{1}-1,
$$

tout terme de la suite (5) étant un terme de la série (4) et tout terme de la série (4) figurant au moins une fois dans la suite (5). Nous pouvons évidemment supposer encore que $m_{1}>1$ et

$$
C_{m_{1}-2}=C_{m_{1}-1}
$$

(puisque'on n'aurait en cas contraire qu'à répéter le dernier terme de la suite (5)).

Nous allons définir maintenant une suite de nombres naturels $m_{1}, m_{2}, m_{3}, \ldots$ et les sous-continus de $C$

$$
C_{k_{1}, k_{2}, \ldots, k_{n}},
$$

où $k_{i}=0,1, \ldots, m_{i}-1, i=1,2, \ldots, n$ et $n=1,2,3, \ldots$, de diamètre 


$$
d\left(C_{k_{1}, k_{2}, \ldots, k_{n}}\right)<1 / 2^{n}
$$

et tels que l'on ait:

$$
C_{k_{1}, k_{2}, \ldots, k_{n-1}} \mathrm{C}
$$

$\left.C C_{k_{1}, k_{2}, \ldots, k_{n-1}, 0}+C_{k_{1}, k_{2}, \ldots, k_{n-1}, 1}+\ldots+C_{k_{1}, k_{2}, \ldots, k_{n-1}, m_{n}-1}{ }^{1}\right)$,

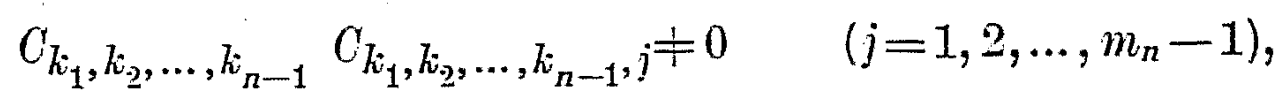

$$
C_{k_{1}, k_{2}, \ldots, k_{n}} C_{\overline{k_{1}}, k_{2}, \ldots, k_{n}} \neq 0 \quad\left(k_{i}=0,1, \ldots, m_{i}-1 ; i=1,2, \ldots, n\right),
$$

${\overline{k_{1}}, k_{2}, \ldots, k_{n}}_{n}$ désignant le système qui suit au système $k_{1}, k_{2}, \ldots, k_{n-1}$ (lorsqu'on range tous les systèmes $k_{11}, k_{2}, \ldots, k_{n}$ selon le principe de premières différences $\left.{ }^{2}\right)$ ) et $\overline{m_{1}-1, m_{2}-1, \ldots, m_{n}-1}$ ayant la même signification que le système $m_{1}-1, m_{2}-1, \ldots, m_{n}-1$. Désignons encore par $k_{1}, k_{2}, \ldots, k_{n}$ le système qui précède le système $k_{1}, k_{2}, \ldots, k_{n}$, lorsqu'on range tous les systèmes $k_{1}, k_{2}, \ldots, k_{n}$ selon le principe de premières différences.

Supposons que les nombres $m_{1}, m_{2}, \ldots, m_{n}$ et les continus (7), contenus dans $G$ et satisfaisant aux conditions (8), (9), (10) et (11), soient définis pour une valeur naturelle de $n$ (ce qui a lieu pour $n=1$ ).

Par hypothèse, le continu $C$ peut être regardé comme une somme $C=G_{1}+G_{2}+\ldots+G_{r}$, où

$$
G_{1}, G_{2}, \ldots, G_{r}
$$

sont des continus de diamètre $<1 / 2^{n+1}$.

Etant donnés les indices $k_{1}, k_{2}, \ldots, k_{n}$ (où $k=0,1, \ldots, m_{i}-1$ et $i=1,2, \ldots, n)$, soient

$$
B_{k_{1}, k_{2}, \ldots, k_{n}}^{\prime}, \quad B_{k_{1}, k_{2}, \ldots, k_{n}}^{\prime \prime}, \ldots, \quad B_{k_{1}, k_{2}, \ldots, k_{n}}^{(I)}
$$

ceux des continus (12) qui ont des points communs avec le continu $C_{k_{1}, k_{2}, \ldots, k_{n}}$. Posons

(14) $S_{k_{1}, k_{2}, \ldots, k_{n}}=B_{k_{1}, k_{2}, \ldots, k_{n}}^{\prime}+B_{k_{1}, k_{2}, \ldots, k_{n}}^{\prime \prime}+\ldots+B_{k_{1}, k_{2}, \ldots, k_{n}}^{(l)}$

1) $C_{k_{1}, k_{2}, \ldots, k_{n-1}}$ pour $n=1$ désignant le continu $C$.

2) c. àd. que de deux systèmes où premier terme par lequel ils diffèrent est le $p$-ième, on écrira d'abord celui des deux systèmes dont $p$-ième terme est plus petit. 
Il résulte de la définition des ensembles (13) et de la formule (14) que $S_{k_{1}, k_{2}, \ldots, k_{n}}$ contient le continu $C_{k_{1}}, k_{2}, \ldots, k_{n}$. Comme $B_{k_{1}, k_{2}, \ldots, k_{n}}^{(i)} C_{k_{1}, k_{2}, \ldots, k_{n}} \neq 0$ pour $i=1,2, \ldots, l$, nous en concluons en vertu du lemme III que $S_{k_{1}}, k_{2}, \ldots, k_{n}$ est un continu.

Soit $p_{k_{1}, k_{2}, \ldots, k_{n}}$ un point commun des ensembles $C_{k_{1}, k_{2}, \ldots, k_{n}}$ et $C_{k_{1}, k_{2}, \ldots, k_{n}}$ (un tel point existe pour $k=0,1, \ldots, m_{i}-1$ et $i=1,2, \ldots, n$ d'après (11)). Posons $p_{0,0, \ldots, 0}=p_{0,0, \ldots, 0}$. Nous aurons done pour $k=0,1, \ldots, m_{i}-1$ et $i=1,2, \ldots, n$ :

$$
p_{k_{1}, k_{2}, \ldots, k_{n}} \subset C_{k_{1}, k_{2}, \ldots, k_{n}}, \quad p_{k_{1}, k_{2}, \ldots, k_{n}} \subset C_{k_{1}, k_{2}, \ldots, k_{n}}
$$

Comme $C_{k_{1}, k_{2}, \ldots, k_{n}} \subset S_{k_{1}}, k_{2}, \ldots, k_{n}$, nous concluons en vertu de (14) et (15) qu'un au moins des termes de la série (14), p. ex. $B_{k_{1}, k_{2}, \ldots, k_{n}}^{\left(u_{k_{1}}, k_{2}, \ldots, k_{n}\right)}$, contient le point $p_{k_{k_{1}, k_{2}, \ldots, k_{n}}}$ et un au moins, p. ex. $B_{k_{1}, k_{2}, \ldots, k_{n}}^{\left(v_{k_{1}}, k_{2}, \ldots, k_{n}\right)}$, contient le point $p_{k_{1}, k_{2}, \ldots, k_{n}}$. En vertu de (14) et du lemme II, il existe donc une chaîne finie de continus

$$
D_{k_{1}, k_{2}, \ldots, k_{n}}^{(0)}, \quad D_{k_{1}, k_{2}, \ldots, k_{n}}^{(1)}, \ldots, \quad D_{k_{1}, k_{2}, \ldots, k_{n}}^{\left(h k_{k_{1}}, k_{2}, \ldots, k_{n}-1\right)}
$$

telle que:

$$
D_{k_{1}, k_{2}, \ldots, k_{n}}^{(0)}=B_{k_{1}, k_{2}, \ldots, k_{n}}^{\left(u k_{k_{1}}, k_{2}, \ldots, k_{n}\right)}, \quad D_{k_{1}, k_{2}, \ldots, k_{n}}^{\left(h_{k_{1}}, k_{2}, \ldots, k_{n}^{-1}\right)}=B_{k_{k_{1}}, k_{2}, \ldots, k_{n}}^{\left(v_{k_{1}}, k_{2}, \ldots, k_{n}\right)}
$$

(18) $D_{k_{1}, k_{2}, \ldots, k_{n}}^{(i)} \cdot D_{k_{1}, k_{2}, \ldots, k_{n}}^{(i+1)} \neq 0 \quad$ pour $i=0,1, \ldots, h_{k_{1}, k_{2}, \ldots, k_{n}}-2$, tout terme de la suite (16) étant égal à un terme de la série (14) et tout terme de la série (14) figurant au moins une fois dans la suite (16).

$\Pi$ est clair que (sauf peut-être le cas $k_{i}=m_{i}-1, i=1,2, \ldots, n$ ) nous avons encore

$$
D_{k_{1}, k_{2}, \ldots, k_{n}}^{\left(h_{k_{1}}, k_{2}, \ldots, k_{n}^{-1}\right)} D_{k_{1}, k_{2}, \ldots, k_{n}}^{(0)} \neq 0
$$

puisque, d'après (17), les deux ensembles du membre gauche contiennent le point $p_{k_{1}, k_{2}, \ldots, k_{n}}$. 
Soit $m_{n+1}$ le plus grand des nombres $h_{k_{1}, k_{2}, \ldots, k_{n}}+1$ où $k_{i}=0,1, \ldots, m_{i}-1$ et $i=1,2, \ldots, n$. Posons:

$$
C_{k_{1}, \ldots, k_{n}, k_{n+1}}=\left\{\begin{array}{l}
D_{k_{1}, k_{2}, \ldots, k_{n}}^{\left(k_{n+1}\right)} \quad \operatorname{pour} \mid \begin{array}{l}
k_{i}=0,1, \ldots, m_{i}-1, i=1,2, \ldots, n \\
\mid k_{n+1}=0,1, \ldots, h_{k_{1}}, k_{2}, \ldots, k_{n}-1,
\end{array} \\
D_{k_{1}, k_{2}, \ldots, k_{n}}^{h_{k_{1}}, k_{2}, \ldots, k_{n}^{-1}} \operatorname{pour} \mid k_{i}=0,1, \ldots, m_{i}-1, i=1,2, \ldots, n \\
h_{k_{1}, k_{2}, \ldots, k_{n} \leqslant k_{n+1} \leqslant m_{n+1}-1 .}, 1
\end{array}\right.
$$

Nous aurons $m_{n+1}-2 \geqslant h_{k_{1}, k_{2}, \ldots, k_{n}}-1$, d'où selon (20) (pour $n$ naturel):

$$
C_{k_{1}, k_{2}, \ldots, k_{n}, m_{n+1}-1}=C_{k_{1}, k_{2}, \ldots, k_{n}, m_{n+1}-2},
$$

ce qui est encore vrai d'après (6) pour $n=0$.

Les formules (20) montrent que tout ensemble

$$
C_{k_{1}, \ldots, k_{n}, k_{n+1}} \text { où } \quad k_{i}=0,1, \ldots, m_{i}-1 \quad \text { et } i=1,2, \ldots, n+1
$$

est égal à un (au moins) des termes de la suite (16) et réciproquement; tout ensemble (22) est donc égal à un (au moins) des termes de la série (14) et réciproquement. Il s'en suit que:

$$
\begin{aligned}
& C_{k_{1}, k_{2}, \ldots, k_{n}} C_{k_{1}, \ldots, k_{n}, k_{n+1}} \neq 0 \\
& \text { pour } k_{i}=0,1, \ldots, m_{i}-1 \text { et } i=1,2, \ldots, n+1 \text {, } \\
& \text { (24) } \quad C_{k_{1}, k_{2}, \ldots, k_{n}} C C_{k_{1}, \ldots, k_{n}, 0}+C_{k_{1}, \ldots, k_{n}, 1}+\ldots+C_{k_{1}, \ldots, k_{n}, m_{n+1}-1} \text {, }
\end{aligned}
$$
et que tout ensemble (22) est un $G_{i}(i=1,2, \ldots, r)$ donc un continu de diamètre $<1 / 2^{n+1}$, contenu dans $C$.

Nous avons éridemment:

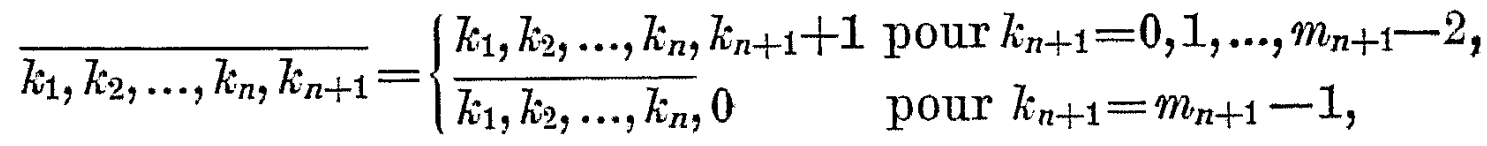
d'où selon $(20)$ :

$$
\begin{aligned}
& C_{k_{1}, \ldots, k_{n}, k_{n+1}}=C_{k_{1}, \ldots, k_{n}, k_{n+1}+1}=D_{k_{1}, k_{2}, \ldots, k_{n}}^{\left(k_{n+1}+1\right)} \\
& \text { pour } k_{n+1}=0,1, \ldots, h_{k_{1}, \ldots, k_{n}}-2 \text {, } \\
& C_{\overline{k_{1}, \ldots, k_{n}, k_{n+1}}}=C_{k_{1}, \ldots, k_{n}, k_{n+1}+1}=D_{k_{k_{1}}, k_{2}, \ldots, k_{n}}^{\left(h_{k_{1}, \ldots, k_{n}}-1\right)} \\
& \text { pour } h_{k_{1}, \ldots, k_{n}}-1 \leqslant k_{n+1} \leqslant m_{n+1}-2 \text {, } \\
& C_{\overline{k_{1}, \ldots, k_{k_{n}}, k_{n+1}}}=C_{\overline{k_{1}, k_{2}, \ldots, k_{n}, 0}}=D{\overline{\bar{k}_{1}, k_{2}, \ldots, k_{n}}}_{-}^{(0)}
\end{aligned}
$$

pour $k_{n+1}=m_{n+1}-1$. 
D'après (18), (19) et (20), nous pouvons donc écrire:

(25) $C_{k_{1}, k_{2}, \ldots, k_{n}, k_{n+1}} \cdot C_{\overline{k_{1}}, k_{k_{2}}, \ldots, k_{n}, k_{n+1}} \neq 0$

pour $k_{i}=0,1, \ldots, m_{i}-1$ et $i=1,2, \ldots, n+1$,

sauf peut-être dans le cas où $k_{i}=m_{i}-1, i=1,2, \ldots, n+1$, dans lequel on a cependant par définition $k_{1}, k_{2}, \ldots, k_{n+1}=k_{1}, k_{2}, \ldots, k_{n+1}$, de sorte que la formule (25) subsiste encore.

Les formules (23), (24) et (25) expriment évidemment les propriétés (10), (9) et (11) pour $n+1$.

La suite infinie des nombres $m_{1}, m_{2}, m_{3}, \ldots$ et les continus (7) contenus dans $C$ et satisfaisant aux conditions (8), (9), (10) et (11) peuvent donc être définis par induction.

Soit

$$
k_{1}, k_{2}, k_{3}, \ldots
$$

une suite infinie donnée d'entiers satisfaisant à l'inégalité

$$
0 \leqslant k_{n} \leqslant m_{n}-1 \quad \text { où } n=1,2,3, \ldots
$$

$\mathrm{Je}$ dis que la suite infinie des continus

$$
C_{k_{1}}, \quad C_{k_{1}, k_{2}}, \quad C_{k_{1}, k_{2}, k_{3}}, \ldots
$$

converge vers un point $p=p\left(k_{1}, k_{2}, k_{3}, \ldots\right)$.

Soit $p_{n}$ un point du continu $O_{k_{1}, k_{2}, \ldots, k_{n}}$. D'après (10), on a

$$
C_{k_{1}, k_{2}, \ldots, k_{n}} C_{k_{1}, k_{2}, \ldots, k_{n}, k_{n+1}} \neq 0 \text {. }
$$

Les diamètres des continus $C_{k_{1}, k_{2}, \ldots, k_{n}}$ et $C_{k_{1}, k_{2}, \ldots, k_{n}, k_{n+1}}$, dont le premier contient le point $p_{n}$ et le second le point $p_{n+1}$, étant (d'après (8)) respectivement $\leqslant 1 / 2^{n}$ et $\leqslant 1 / 2^{n+1 \cdot 1}$, il vient

$$
\varrho\left(p_{n}, p_{n+1}\right) \leqslant \frac{1}{2^{n}}+\frac{1}{2^{n+1}}=\frac{3}{2^{n+1}},
$$

$\varrho\left(p_{n}, p_{n+1}\right)$ désignant la distance entre $p_{n}$ et $p_{n+1}$.

L'inégalité (29) donne (pour $q$ naturel):

$$
\varrho\left(p_{n}, p_{n+q}\right) \leqslant \frac{3}{2^{n+1}}+\frac{3}{2^{n+2}}+\ldots+\frac{3}{2^{n+q}}<\frac{3}{2^{n}},
$$

ce qui montre que la suite $\left\{p_{n}\right\}$ converge vers un point limite 
$p=\lim _{n \rightarrow \infty} p_{n^{*}}$. Or, l'inégalité (30) donne pour $q \rightarrow \infty$

$$
\varrho\left(p_{n}, p\right) \leqslant 3 / 2^{n} .
$$

Le diamètre de $C_{k_{1}, k_{2}, \ldots, k_{n}}$ étant $\leqslant 1 / 2^{n}$ et $p_{n}$ appartenant à $C_{k_{1}}, k_{2} \ldots, k_{n}$, on conclut de (31) que tout point de l'ensemble $C_{k_{1}}, k_{2}, \ldots, k_{n}$ est à une distance $\leqslant t / 2^{n}$ de $p$, ce qui montre que la suite (28) converge vers le point $p$.

A toute suite infinie (26) d'entiers satisfaisant aux inégalités $(27)$ correspond donc un point $p=p\left(k_{1}, k_{2}, k_{3}, \ldots\right)$ qui est un point limite des continus (28).

Soit maintenant $t$ un nombre réel donné tel que

$$
0 \leqslant t<1 \text {. }
$$

Posons ( $E$ désignant l',entier de"):

$$
\begin{gathered}
k_{1}=E m_{1} t \\
k_{n}=E m_{1} m_{2} \ldots m_{n} t-m_{n} E m_{1} m_{2} \ldots m_{n-1} t \quad \text { pour } n=2,3,4, \ldots
\end{gathered}
$$

Nous aurons évidemment d'après (32) et (33):

$$
\begin{gathered}
0 \leqslant k_{1} \leqslant m_{1}-1 \\
m_{1} m_{2} \ldots m_{n} t-1<E m_{1} m_{2} \ldots m_{n} t \leqslant m_{1} m_{2} \ldots m_{n} t \\
m_{1} m_{2} \ldots m_{n-1} t-1<E m_{1} m_{2} \ldots m_{n-1} t \leqslant m_{1} m_{2} \ldots m_{n-1} t
\end{gathered}
$$

donc $-1<E m_{1} m_{2} \ldots m_{n} t-m_{n} E m_{1} m_{2} \ldots m_{n-1} t<m_{n}$, c'est à dire $0 \leqslant E m_{1} m_{2} \ldots m_{n} t-m_{n} E m_{1} m_{2} \ldots m_{n-1} t \leqslant m_{n}-1$, d'où en vertu de (33)

$$
0 \leqslant k_{n} \leqslant m_{n}-1 \quad \text { pour } \quad n=2,3,4, \ldots
$$

Nous pourons donc écrire, d'après (34) et (35)

$$
0 \leqslant k_{n} \leqslant m_{n}-1 \quad \text { pour } \quad n=1,2,3, \ldots .
$$

A tout nombre réel $t$ satisfaisant à l'inégalité (32) correspond par conséquent une suite d'entiers (26) satisfaisant à l'inégalité (27), donc aussi un point limite de la suite infinie des continus (28)

$$
p(t)=p\left(k_{1}, k_{2}, k_{3}, \ldots\right) .
$$


Posons encore

$$
p(1)=\lim _{n \rightarrow \infty} C_{m_{1}-1, m_{2}-1, \ldots, m_{n}-1} .
$$

Nous allons montrer que $p(t)$ est une fonction continue de $t$ pour $0 \leqslant t \leqslant 1$.

Soient dans l'intervalle $\langle 0,1\rangle t^{\prime}, t^{\prime \prime}$ deux nombres tels que $t^{\prime \prime}<1$ et

$$
0 \leqslant t^{\prime \prime}-t^{\prime} \leqslant 1 / m_{1} m_{2} \ldots m_{q}
$$

et soient $\left\{k_{n}^{\prime}\right\},\left\{k_{n}^{\prime \prime}\right\}$ les suites correspondantes des $k_{n}$, déterminées d'après les formules (33), en y remplaçant respectivement $t$ par $t^{\prime}$ et $t^{\prime \prime}$.

Supposons que pour un indice $i<q$

$$
E m_{1} m_{2} \ldots m_{i} t^{\prime \prime}=E m_{1} m_{2} \ldots m_{i} t^{\prime \prime}
$$

D'après (37), nous aỵons

$$
0 \leqslant m_{1} m_{2} \ldots m_{i} t^{\prime \prime}-m_{1} m_{2} \ldots m_{i} t^{\prime} \leqslant m_{1} m_{2} \ldots m_{q} / m_{1} m_{2} \ldots m_{q} \leqslant 1
$$

d'où

$$
0 \leqslant E m_{1} m_{2} \ldots m_{i} t^{\prime \prime}-E m_{1} m_{2} \ldots m_{i} t^{\prime} \leqslant 1
$$

ce qui donne d'après (38) l'égalité

$$
E m_{1} m_{2} \ldots m_{i} t^{\prime \prime}=E m_{1} m_{2} \ldots m_{i} t^{\prime}+1 \text {. }
$$

Or, comme on voit sans peine, l'inégalité (38) entraîne à plus forte raison l'inégalité

$$
E m_{1} m_{2} \ldots m_{i} m_{i+1} t^{\prime} \neq E m_{1} m_{2} \ldots m_{i} m_{i+1} t^{\prime \prime}
$$

donc aussi l'égalité

$$
E m_{1} m_{2} \ldots m_{i+1} t^{\prime \prime}=E m_{1} m_{2} \ldots m_{i+1} t^{\prime}+1 \text {. }
$$

Soit $s<q$ le plus petit indice pour lequel

$$
E m_{1} m_{2} \ldots m_{s} t^{\prime} \neq E m_{1} m_{2} \ldots m_{s} t^{\prime \prime} \text {. }
$$


Il vient

$$
E m_{1} m_{2} \ldots m_{i} t^{\prime \prime}= \begin{cases}E m_{1} m_{2} \ldots m_{i} t^{\prime} & \text { pour } \quad i=1,2, \ldots, s-1, \\ E m_{1} m_{2} \ldots m_{i} t^{\prime}+1 & \text { pour } \quad i=s, s+1, \ldots, q,\end{cases}
$$
d'où selon (33):

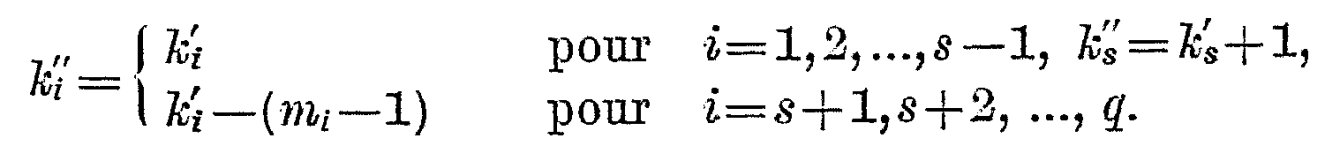

Or, cette dernière formule montre d'après (35) que

$k_{i}^{\prime}=m_{i}-1 \quad$ et $\quad k_{i}^{\prime \prime}=0$ pour $i=s+1, s+2, \ldots, q$.

Les systèmes $k_{1}^{\prime}, k_{2}^{\prime}, \ldots, k_{q}^{\prime}$ et $k_{1}^{\prime \prime}, k_{2}^{\prime \prime}, \ldots, k_{q}^{\prime \prime}$ coïncident donc respectivement avec les systèmes

et

$$
k_{1}^{\prime}, k_{2}^{\prime}, \ldots, k_{s-1}^{\prime}, k_{s}^{\prime}, m_{s+1}-1, m_{s+2}-1, \ldots, m_{q}-1
$$

$$
k_{1}^{\prime}, k_{2}^{\prime}, \ldots k_{s-1}^{\prime}, k_{s}^{\prime}+1,0,0, \ldots, 0 \text {. }
$$

Par conséquent $k_{1}^{\prime \prime}, k_{2}^{\prime \prime}, \ldots, k_{q}^{\prime \prime}=\overline{k_{1}^{\prime}, k_{2}^{\prime}, \ldots, k_{q}^{\prime}}$, d'où selon (11)

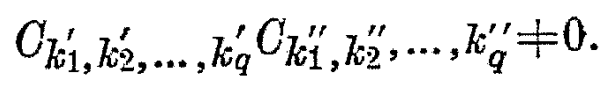

Dans le cas où l'inégalité (38) ne se présente pour aucun indice $i \leqslant q$, nous arons

$$
E m_{1} m_{2} \ldots m_{i} t^{\prime}=E m_{1} m_{2} \ldots m_{i} t^{\prime \prime} \quad \text { pour } \quad i=1,2, \ldots, q,
$$

d'où, selon (33), $k_{i}^{\prime}=k_{i}^{\prime \prime}$ pour $i=1,2, \ldots, q$. Les systèmes

$$
k_{1}^{\prime}, k_{2}^{\prime}, \ldots, k_{q}^{\prime} \quad \text { et } \quad k_{1}^{\prime \prime}, k_{2}^{\prime \prime}, \ldots, k_{q}^{\prime \prime}
$$

sont donc identiques et la formule (40) subsiste encore.

Nous avons ainsi démontré que l'inégalité (37) entraîne toujours la formule $(40)$ pour $t^{\prime \prime}<1$.

Soit maintenant $t^{\prime \prime}=1$ et $0 \leqslant t^{\prime}<t$. L'inégalité (37) devient alors

$$
1-\frac{1}{m_{1} m_{2} \ldots m_{q}} \leqslant t^{\prime}<1,
$$

ce qui donne pour $i=1,2, \ldots, q$

d'où

$$
m_{1} m_{2} \ldots m_{i}-\frac{1}{m_{i+1} \ldots m_{q}} \leqslant m_{1} m_{2} \ldots m_{i} t^{i}<m_{1} m_{2} \ldots m_{i},
$$

$$
E m_{1} m_{2} \ldots m_{i} t^{\prime}=m_{1} m_{2} \ldots m_{i}-1
$$


donc, d'après (33),

$$
k_{i}^{\prime}=m_{i}-1 \quad \text { pour } \quad i=1,2, \ldots, q .
$$

D'après (36), nous pouvons regarder la suite des $k_{n}^{\prime \prime}=m_{n}-1$ $(n=1,2,3, \ldots)$ comme correspondant ̀े $t^{\prime \prime}=1$; nous avons donc d'après (41)

$$
k_{i}^{\prime}=k_{i}^{\prime \prime} \quad \text { pour } \quad i=1,2, \ldots, q
$$

et la formule (40) subsiste encore.

Nous avons ainsi démontré que l'inégalité (37) entraîne la formule $(40)$ pour deux nombres quelconques $t^{\prime}$ et $t^{\prime \prime}$ de l'intervalle $\langle 0,1\rangle$.

Soit $p_{q}^{\prime}$ un point de $C_{k_{1}^{\prime}, k_{2}^{\prime}, \ldots, k_{q}^{\prime}}$ et $p_{q}^{\prime \prime}$ un point de $C_{k_{1}^{\prime \prime}, k_{2}^{\prime \prime}, \ldots, k_{q}^{\prime \prime}}$

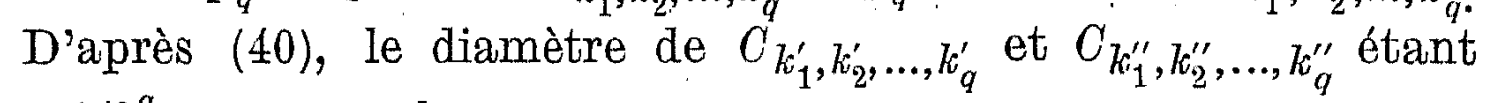
$<1 / 2^{q}$, nous concluons que

$$
\varrho\left(p_{q}^{\prime}, p_{q}^{\prime \prime}\right)<2 / 2^{q} .
$$

Or, soit $p^{\prime}=p\left(t^{\prime}\right)$ la limite de la suite des continus

$$
C_{k_{1}^{\prime}}, \quad C_{k_{1}^{\prime}, k_{2}^{\prime}}, \quad C_{k_{1}^{\prime}, k_{2}^{\prime}, k_{3}^{\prime}}, \ldots
$$

et $p^{\prime \prime}=p\left(t^{\prime \prime}\right)$ celle de la suite des continus

$$
C_{k_{1}^{\prime \prime}}, \quad C_{k_{1}^{\prime \prime}, k_{2}^{\prime \prime}}, \quad C_{k_{1}^{\prime \prime}, k_{2}^{\prime \prime}, k_{3}^{\prime \prime}}, \ldots
$$

Nous avons démontré plus haut (p. 51) que tout point de $C_{k_{1}, k_{2}, \ldots, k_{n}}$ est à une distance $<4 / 2^{n}$ du point $\lim _{n \rightarrow \infty} C_{k_{1}}, k_{2}, \ldots, k_{n}$. Done:

$$
\varrho\left(p_{q}^{\prime}, p^{\prime}\right)<4 / 2^{q}, \quad \varrho\left(p_{q}^{\prime \prime}, p^{\prime \prime}\right)<4 / 2^{q},
$$

et d'après $(42)$

$$
\varrho\left(p\left(t^{\prime}\right), p\left(t^{\prime \prime}\right)\right)=\varrho\left(p^{\prime}, p^{\prime \prime}\right)<10 / 2^{q} .
$$

Il est ainsi établi que l'inégalité (37) entraîne (pour $0 \leqslant t^{\prime} \leqslant 1$ et $0 \leqslant t^{\prime \prime} \leqslant 1$ ) l'inégalité (43), ce qui montre que la fonction $p(t)$ est continue pour $0 \leqslant t \leqslant 1$. 
Il s'en suit de la définition de la fonction $p(t)$ que, pour tout nombre $t$ satisfaisant à l'inégalité $0 \leqslant t \leqslant 1$, le point $p(t)$ est un point d'accumulation de l'ensemble $C$, donc ( $C$ étant un continu) un point de $C$. Or, nous allons montrer que, pour tout point $p_{0}$ de $C$, il existe au moins une valeur de $t(0 \leqslant t \leqslant 1)$ telle que $p(t)=p_{0}$.

Soit, en effet, $p_{0}$ un point donné de $C$. D'après (9)

$$
C \subset C_{0}+C_{1}^{n}+\ldots+C_{m_{1}-1} \text {. }
$$

Le point $p_{0}$ appartenant à $C$, il appartient à un au moins des ensembles $C_{l}$ où $l=0,1,2, \ldots, m_{1}-1$. Soit $l_{1}$ le plus petit indice pour lequel $p_{0} \subset C_{l_{1}}$. D'après (21), on a $l_{1} \leqslant m_{1}-2$ et d'après (9)

$$
C_{l_{1}} \subset C_{l_{1}, 1}+C_{l_{1}, 2}+\ldots+C_{l_{1}, m_{z}-1} \text {. }
$$

En raisonnant comme auparavant, nous en concluons qu'il existe un indice $l\left(0 \leqslant l \leqslant m_{2}-2\right)$ tel que $p_{0} \subset C_{l_{1},}$; soit $l_{2}$ le plus petit indice $l$ satisfaisant à cette condition. En répétant ce raisonnement, nous arrivons à une suite infinie de continus

$$
C_{l_{1}}, \quad C_{l_{1}, l_{2}}, \quad C_{l_{1}, l_{2}, l_{3}}, \ldots \quad\left(l_{i}=0,1, \ldots, m_{i}-2\right)
$$

telle que

$$
p_{0} \subset C_{l_{1}}, l_{2}, \ldots, l_{n} \quad(n=1,2, \ldots) .
$$

Posons maintenant

$$
t_{0}=\frac{l_{1}}{m_{1}}+\frac{l_{2}}{m_{1} m_{2}}+\frac{l_{3}}{m_{1} m_{2} m_{3}}+\ldots
$$

Comme

$$
0 \leqslant l_{n} \leqslant m_{n}-2
$$$$
(n=1,2, \ldots),
$$

nous concluons sans peine que la série (46) converge et que $0 \leqslant t_{0}<1$.

Les formules (33) donnent pour $t=t_{0}$, d'après (46) et (47):

$$
k_{n}=l_{n}
$$$$
(n=1,2, \ldots) \text {. }
$$

Or, en déterminant les nombres $k_{n}$ des formules (33), nous avons pour $0 \leqslant t<1$

$$
p(t)=\lim _{n \rightarrow \infty} C_{k_{1}, k_{2}, \ldots, k_{n},}
$$


donc, d'après (48), pour $t=t_{0}$

$$
p\left(t_{0}\right)=\lim _{n \rightarrow \infty} C_{l_{1}}, l_{2}, \ldots, l_{n l}
$$

Les formules $(45)$ et $(49)$ donnent sans peine $p_{0}=p\left(t_{0}\right)$. Nous avons ainsi démontré que l'ensemble $C$ coïncide avec l'ensemble de tous les points

$$
(0 \leqslant t \leqslant 1)
$$

La fonction $p(t)$ étant continue pour $0 \leqslant t \leqslant 1$, il en résulte que $C$ est une image univoque et continue de l'intervalle $\langle 0,1\rangle$ : c'est donc une courbe jordanienne.

Nous arons ainsi établi que la condition de notre théorème est suffisante; nous allons montrer maintenant qu'elle est nécessaire.

Soit $C$ une courbe jordanienne. Il existe donc une fonction $p(t)$ continue pour $0 \leqslant t \leqslant 1$ et telle que l'ensemble $C$ coïncide avec l'ensemble de tous les points $(50)$.

Soit $\varepsilon$ un nombre positif donné. La fonction $p(t)$ étant continue pour $0 \leqslant t \leqslant 1$, il existe un entier positif $n$ tel que l'inégalité $\left|t-t^{\prime}\right|<1 / n$ entraîne pour $t$ et $t^{\prime}$ de l'intervalle $\langle 0,1\rangle$ l'inégalité $\varrho\left(p(t), p\left(t^{\prime}\right)\right)<\varepsilon$.

Désignons par $C_{k}$ l'ensemble de tous les points $p(t)$ pour lesquels

$$
(k-1) / n \leqslant t \leqslant k / n
$$

Nous aurons évidemment $C=C_{1}+C_{2}+\ldots+C_{n}$ et $C_{k}(k=1,2, \ldots, n)$ seront des continus de diamètre $<\varepsilon$. La condition de notre théorème est donc nécessaire, c. q.f. d.

Notre théorème étant ainsi établi, nous allons l'appliquer à démontrer un théorème de M. Mazurkiewicz sur les continus jordaniens.

Soit $C$ un continu donné. Appelons point de premier genre de $C$ tout point $p$ tel que, pour tout nombre donné $\varepsilon>0$, il existe un sous-continu $K$ de $C$ de diamètre $<\varepsilon$ et un nombre $\delta>0$ tels que tout point de $O$ dont la distance du point $p$ 
est $<\delta$ est contenu dans $k$. On peut démontrer sans peine que cette définition équivaut à la définition du point de premier genre d'un continu, donnée par M. Mazurkiewicz ${ }^{4}$ ).

Le théorème de M. Mazurkiewicz s'exprime comme il suit $\left.{ }^{5}\right)$ :

Pour qu'un continu $C$ soit une courbe jordanienne, it faut et il suffit que tous les points de $C$ soient de premier genre.

Démonstration. Soient (dans l'espace euclidien à $m$ dimensions): $C$ un continu jordanien, $\varepsilon$ un nombre positif quelconque, $p$ un point donné de $C$. Il existe en vertu de notre théorème une décomposition

$$
C=C_{1}+C_{2}+\ldots+C_{n}
$$

où $C_{i}(i=1,2, \ldots, n)$ sont des continus de diamètre $<\varepsilon / 2$. Comme $p$ appartient à $C$, un au moins des $C_{i}$ contient $p$. Soient

$$
C q_{1}, C q_{2}, \ldots, C q_{k}
$$

ceux parmi les termes de (51) qui contiennent $p$ et

$$
C_{r_{1}}, C_{r_{2}}, \ldots, C_{r_{l}}
$$

tous les autres termes de cette décomposition (s'il en existe). Posons

$$
K=C q_{1}+C_{q_{2}}+\ldots+C q_{k} .
$$

Chacun des ensembles (52) étant un continu de diamètre $<\varepsilon / 2$, et contenant $p$, on voit sans peine que $K$ est un continu de diamètre $<\varepsilon$.

Or, posons

$$
L=C_{r_{1}}+C_{r_{2}}+\ldots+C_{r_{i}}
$$

C'est donc par définition un ensemble fermé (ou vide) ne contenant pas $p$. Il existe par conséquent un nombre positif $\delta$ tel que tout point de $L$ est situé à distance $\geqslant \delta$ du point $p$. (Dans le cas où l'ensemble $L$ est vide, on peut poser p.ex.

$\left.{ }^{4}\right)$ S. Mazurkiewicz, Sur une classification des points situés sur un continu arbitraire, Comptes Rendus de la Société des Sciences de Varsovie. IX (1916), p. 441.

5) 1. c., p. 442 . 
$\delta=1$ ). Done, tout point de $C$ dont la distance de $p$ est $<\delta$ appartient à $K$ (puisque $K \supset C-L$ ). Nous avons ainsi démontré que $p$ est un point de premier genre (par rapport au continu $C$ ). La condition de M. Mazurkiewicz est donc nécessaire.

Admettons maintenant que tout point du continu $C$ est de premier genre et soit $\varepsilon$ un nombre positif donné.

Envisageons toutes les sphères $m$-dimensionnelles $S$ jouissant des propriétés suivantes:

$1^{0}$ Le centre de la sphère $S$ a des coordonnées rationnelles.

$2^{0}$ Le rayon de $S$ est rationnel.

$3^{0} \mathrm{Il}$ existe un continu de diamètre $<\varepsilon$ contenu dans $C$ et contenant $S C$.

A toute sphère $S$ qui satisfait aux conditions $1^{0}, 2^{0}$ et $3^{0}$, faisons correspondre un continu $C_{S}$ de diamètre $<\varepsilon$ contenu dans $C$ et contenant $S C$; un tel continu $C_{S}$ existe en vertu de $3^{0}$ (quant à la correspondance en question, son existence résulte de l'axiome du choix).

Je dis que tout point $p$ du continu $C$ est intérieur au moins à une sphère $S$ satisfaisant aux conditions $1^{0}, 2^{0}$ et $3^{0}$. En effet, tout point de $C$ étant par hypothèse un point de premier genre, il existe pour $\varepsilon$ considéré, un sous-continu $K$ de $C$ de diamètre $<\varepsilon$ et un nombre positif $\delta$ tels que tous les points de $C$ situés à distance $<\delta$ du point $p$ appartiennent à $K$. Soit $S=S(p)$ une sphère assujettie aux conditions $1^{0}$ et $2^{0}$, de centre situé à distance $<\delta / 2$ du point $p$ et de rayon $r$ compris entre $\delta / 2$ et $\delta$. Tout point de la sphère $S$ est donc à la distance $<\delta$ du point $p$; il en résulte que l'ensemble $S C$ est contenu dans $K$. Par conséquent, la sphère $S$ satisfait aux conditions $1^{0}, 2^{0}$ et $3^{0}$; en même temps, $p$ est un point intérieur de $S$ (comme situé à distance $<\delta / 2<r$ de son centre).

L'ensemble de toutes les sphères qui satisfont aux conditions $1^{0}, 2^{0}$ et $3^{0}$ est done non vide; or, il est évidemment au plus dénombrable et nous pouvons par conséquent les ranger en une suite

$$
S_{1}, S_{2}, S_{3}, \ldots
$$


Si la suite (53) est finie, nous pourons la remplacer par une suite infinie, p. ex. en la répétant périodiquement. Supposons donc que la suite (53) est infinie et contient (au moins une fois) toute sphère $S$ satisfaisant aux conditions $1^{0}, 2^{0}$ et $3^{0}$. Soit

$$
C_{S_{1}}, C_{S_{3}}, C_{S_{3}}, \ldots
$$

la suite infinie de continus $C_{S}$ correspondants situés dans $C$. $J$ e dis qu'il existe un indice $n$ tel que

$$
C=C_{S_{1}}+C_{S_{2}}+\ldots+C_{S_{n}} \text {. }
$$

En effet, en supposant le contraire, il existerait dans $C$, pour tout indice $n$, un point $p_{n}$ qui n'appartient pas à l'ensemble

$$
C_{S_{1}}+C_{S_{2}}+\ldots+C_{S_{n}}
$$

Le continu $C$ étant un ensemble borné, on peut notoirement extraire de toute suite infinie de ses points

$$
p_{1}: p_{2}, p_{3}, \ldots
$$

une suite convergente $p_{n_{1}}, p_{n_{2}}, p_{n_{3}}, \ldots$ où $n_{1}<n_{2}<n_{3}<\ldots$ et, $O$ étant un ensemble fermé, le point

$$
p_{0}=\lim _{k \rightarrow \infty} p_{n_{k}}
$$

appartiendrait encore à $C$.

Or, nous avons établi que tout point de $C$ est intérieur au moins à une sphère $S$ satisfaisant aux conditions $1^{0}, 2^{0}$ et $3^{0}$, donc au moins à une sphère de la suite (53). Soit $S_{q}$ une sphère de la suite (53) contenant dans son intérieur le point $p_{0}$. D'après (58), il existe un indice $n \geqslant q$ tel que le point $p_{n}$ est aussi intérieur à la sphère $S_{q}$. Le point $p_{n}$ appartient donc à $S_{q} C$, et par conséquent à $C_{S_{q}}$ (d'après la définition du continu $\left.\sigma_{S}\right)$. D'autre part, selon la définition de la suite $(57)$, le point $p_{n}$ n'appartient pas à la somme (56), done à plus forte raison à l'ensemble $C_{S_{q}}$, puisque $n \geqslant q$. C'est une contradiction.

L'existence d'un indice $n$ pour lequel on a la formule ( 555$)$ est ainsi établie. Chacun des ensembles (54) étant un continu de diamètre $<\varepsilon$ contenu dans $C$, nous avons donc démontré que $C$ est une somme d'un nombre fini de continus de dia- 
mètre $<\varepsilon$. Comme $\varepsilon$ est un nombre positif arbitraire, il en résulte que le continu $C$ satisfait à la condition de notre théorème: c'est donc une courbe jordanienne. La condition de M. Mazurkiewicz est par conséquent suffisante.

Remarquons encore qu'on pourrait aussi, en s'appuyant sur notre théorème, démontrer sans difficulté un autre théorème de M. Mazurkiewicz ${ }^{6}$ ):

Pour qu'un continu $C$ soit une courbe jordanienne, il faut et il suffit qu'à tout couple $a, b$ de ses points corresponde un continu $C(a, b)$ contenant $a$ et $b$ et contenu dans $C$, le diamètre de $C(a, b)$ tendant vers 0 avec la distance des points a et $b$.

6) S. Mazurkiewicz, Sur l'arithmétisation des continus II, Comptes Rendus de la Société des Sciences de Varsovie VI (1913), p. 945. 